\title{
Evaluation of the apoptosis pathway in tumor and non-tumor cell lines with altered expression of the NEK5 kinase
}

\section{Ana Luisa R. de Oliveira*, Jörg Kobarg.}

\begin{abstract}
NEK5 is a member of the NEK kinase family that participates in cell cycle regulation, and studies have demonstated that NEK5 is a substrate for caspase-3 and is associated with cell death and cell respiration. To better evaluate the relation between this kinase and cell death, the aim of this project is to analyse the role of NEK5 in the apoptosis pathway. For this, the expression of proteins related to this pathway will be quantified by Western Blot in cells expressing NEK5 and in cells depleted for it, in tumor and non-tumor cell lines. The expression of proteins in non-tumor cell lines suggest that the expression of NEK5 is connected to an anti-apoptotic signaling in the cell.
\end{abstract}

\section{Key words:}

NEK5, apoptosis, cell signaling.

\section{Introduction}

NEKs (NIMA-related kinases) are one of the least characterized family of cell cycle kinases. They are commonly associated to three main functions: centrioles and mitosis regulation, primary ciliary functions and DNA damage response. Among all 11 NEKs found in humans, NEK5 is one with little amount of information. It was demonstrated that NEK5 is a substrate for caspase-3 and is involved in skeletal muscle differentiation ${ }^{1 .}$ Moreover, our research group demonstrated that NEK5 is a mitochondrial protein that interacts with Cox11 (member of cytochrome c oxidase) and BCLAF1 (Bcl-2associated transcription factor 1), and participates in the regulation of cell respiration and cell death ${ }^{2}$. In this project, we aim to evaluate the role of the NEK5 kinase in the intrinsic apoptosis pathway in tumor (U2OS) and non-tumor (HEK293T) cell lines. We evaluated the expression of the intrinsic apoptosis signaling proteins by Western Blot of cells with altered expression of NEK5 (silenced and overexpression) as well as cells expressing NEK5 K33A, a "kinase dead" version. As part of the project, we will also generate U2OS Flp-In NEK5 and U2OS FIp-In NEK5 K33A cells.

\section{Results and Discussion}

To generate U2OS Flp-In NEK5 and U2OS FlpIn NEK5 K33A, it was used the Flp-In ${ }^{T M}$ T-REx ${ }^{T M}$ system, that consists of generating stable cell lines with tetracycline-inducible expression of a gene. So far, it was generated a U2OS Flp-In ${ }^{T M} T-R E x^{T M}$ host cell line, through the transfection of the plasmids pFRT/lacZeo and pcDNA6/TR.

Meanwhile, it was performed Western blot for the HEK293T cells. The proteins analyzed, with their expression compared to control, can be seen in charts 1 and 2. Statistical significance was obtained using Student's T-Test, with $\mathrm{p}<0.05$.

Chart 1. Expression of proteins in cells depleted for NEK5.

\begin{tabular}{|c|c|}
\hline & shNEK5 \\
\hline Cytochrome c & $\downarrow(* *)$ \\
\hline Bcl-xL & $\uparrow(* *)$ \\
\hline
\end{tabular}

Chart 2. Expression of proteins in Flp-In NEK5 cells.

\begin{tabular}{|c|c|c|}
\hline & NEK5 & NEK5 K33A \\
\hline AIF & $\downarrow\left(^{* * *}\right)$ & $=$ \\
\hline BAX & $\downarrow\left(^{* *}\right)$ & $\uparrow(* *)$ \\
\hline Caspase-3 & $\downarrow\left(^{*}\right)$ & $=$ \\
\hline Caspase-9 & $\downarrow\left(^{* * *}\right)$ & $\downarrow\left(^{*}\right)$ \\
\hline
\end{tabular}

We can imply that there is a tendency for an anti-apoptotic signaling. This can be observed by a significant decrease of pro-apoptotic proteins (AIF, BAX, caspase-3 and caspase-9) in cells expressing NEK5. Moreover, the cells depleted for NEK5 also show an inclination for anti-apoptotic signaling. Although the results indicate an increase in $\mathrm{Bcl}-\mathrm{xL}$ (pro-survival) and a decrease in cytochrome $c$ (pro-apoptotic) in NEK5 depleted cells, this may be due to an adaptive response of the cell after depletion of NEK5. Therefore, the cell compensates the lack of NEK5 by increasing $\mathrm{Bcl}-\mathrm{xL}$, so NEK5 might have an anti-apoptotic role.

\section{Conclusions}

In conclusion, our data suggest that NEK5 may be related to an anti-apoptotic signaling, mainly because of the decrease of pro-apoptotic proteins found in cells expressing NEK5. It is necessary, in the future, to compare the expression of these proteins with other models, and to evaluate the activity of caspases, since they are synthesized as inactive proteases.

\section{Acknowledgement}

The authors would like to acknowledge Dra. Talita D. Melo Hanchuk for providing the NEK5 depleted HEK293T cells used in this project. This work was supported by Conselho Nacional de Desenvolvimento Científico e Tecnológico (CNPq) and Fundação de Ampara à Pesquisa do Estado de São Paulo (FAPESP).

\footnotetext{
${ }^{1}$ Shimizu, K.; Sawasaki, T. Nek5, a novel substrate for caspase-3, promotes skeletal muscle differentiation by up-regulating caspase activity. FEBS Lett., 587 (2013), pp. 2219-2225.

${ }^{2}$ Hanchuk, T. D. M.; Papa, P. F.; La Guardia, P. G. ; Vercesi, A. E. ; Kobarg, J. Nek5 interacts with mitochondrial proteins and interferes negatively in mitochondrial mediated cell death and respiration. Cell Signal, 27 (2015), pp. 1168-1177.
} 